TRANSACTIONS OF THE

AMERICAN MATHEMATICAL SOCIETY

Volume 350, Number 11, November 1998, Pages 4423-4444

S 0002-9947(98)02355-1

\title{
BORDISM OF SPIN 4-MANIFOLDS WITH LOCAL ACTION OF TORI
}

\author{
PIOTR MIKRUT
}

\begin{abstract}
We prove that bordism group of spin 4-manifolds with singular $T$ structure, the notion introduced by Cheeger and Gromov, is an infinite cyclic group and is detected by singnature. In particular we obtain that the theorem of Atiyah and Hirzebruch of vanishing of $\hat{A}$-genus in case of $S^{1}$ action on spin $4 n$-manifolds is not valid in case of $T$-structures on spin 4-manifolds.
\end{abstract}

\section{INTRODUCTION}

The notion of a local action of tori on a manifold is a generalization of an action of a torus. Tori, possibly of different dimensions, act effectively on open subsets of the manifold and these actions fit together on overlaps in such a way that the torus acting on one of the sets injects homomorphically into the torus acting on the second one. If we assume that each of these actions is without fixed points, then the local action of tori coincides with a $T$-structure. The notion of a $T$-structure and more general notions of an $F$ structure and a nilpotent Killing structure appeared in $[\mathrm{G}],[\mathrm{CG} 1],[\mathrm{CG} 2],[\mathrm{CFG}]$ in the context of collapsing Riemannian manifolds.

Here we assume that the manifold is compact, differentiable, spin and that the local action of tori may admit fixed points. The main result of the paper is

Theorem. The bordism group of compact spin 4-manifolds admitting local actions of tori is isomorphic to $Z$. There is a generator with signature 16 .

In particular we obtain that any compact spin 4-manifold is spin cobordant with a manifold admitting local action of tori. In dimension $4 \operatorname{Sign}(M)=-\frac{1}{8} \hat{A}(M)$, so this theorem gives a counterexample to the extension of the Atiyah and Hirzebruch theorem $[\mathrm{AH}]$ to the case of mixed local actions of tori on compact spin four dimensional manifolds. If we assume that the local action of tori is pure (i.e. the dimensions of the acting tori are all equal) the following problem remains open:

Conjecture. $\hat{A}(M)=0$ for a compact spin $4 n$-manifold admitting pure local action of tori.

According to the present knowledge of the author the statement of the conjecture is true for pure local action of tori on 4-manifolds and under certain assumptions on the dimension of the acting torus for 8-manifolds.

Received by the editors June 25, 1996.

1991 Mathematics Subject Classification. Primary 57M60, 57N13, 57R15, 57R20, 57R85.

Key words and phrases. T-structure, bordism, spin manifold, 4-manifold, signature.

The author was partially supported by the Polish Commitee of Scientific Research grant $4241 / \mathrm{PB} / \mathrm{IM} / 95$.

(C)1998 American Mathematical Society 
A proof of the statement in the case of dimension 4 and local $T^{2}$ actions can be found in the authors thesis [Mi1]. It can also be deduced from the results of the present paper.

I would like to thank Marek Lewkowicz, who patiently explained to me the subtleties of the notion of the $T$-structure; Tadeusz Januszkiewicz, who encouraged me to address the problem.

Definition 0.1. We say that a smooth manifold $M$ admits a local action of tori if there is a covering $\left\{U_{\alpha}\right\}_{\alpha \in \Lambda}$ of $M$ by open sets such that for each $\alpha \in \Lambda$ there is a torus $T^{k_{\alpha}}$ which acts smoothly and effectively on $U_{\alpha}$ :

$$
\theta_{\alpha}: T^{k_{\alpha}} \times U_{\alpha} \rightarrow U_{\alpha}
$$

and if $U_{\alpha \beta}:=U_{\alpha} \cap U_{\beta} \neq \emptyset$, then up to changing the role of $\alpha, \beta$ we have $k_{\alpha} \leq k_{\beta}$ and there exists a monomorphism

$$
\xi_{\alpha \beta}: T^{k_{\alpha}} \rightarrow T^{k_{\beta}}
$$

such that the following diagram is commutative:

$$
\begin{aligned}
T^{k_{\alpha}} \times U_{\alpha \beta} & \stackrel{\theta_{\alpha}}{\longrightarrow} U_{\alpha \beta} \\
\xi_{\alpha \beta} \times i d_{U_{\alpha \beta}} \downarrow & i d_{U_{\alpha \beta}} \downarrow \\
T^{k_{\beta}} \times U_{\alpha \beta} & \stackrel{\theta_{\beta}}{\longrightarrow} U_{\alpha \beta}
\end{aligned}
$$

Moreover if $U_{\alpha} \cap U_{\beta} \cap U_{\gamma} \neq \emptyset$, then $\xi_{\beta \gamma} \xi_{\alpha \beta}=\xi_{\alpha \gamma}\left(k_{\alpha} \leq k_{\beta} \leq k_{\gamma}\right)$.

An atlas of the local action of tori is the collection of the sets and maps:

$$
\left\langle\left\{U_{\alpha}\right\}_{\alpha \in \Lambda} ;\left\{\theta_{\alpha}\right\}_{\alpha \in \Lambda} ;\left\{\xi_{\alpha \beta}\right\}_{(\alpha, \beta) i) \Lambda_{0}}\right\rangle .
$$

Here $\Lambda_{0}$ is the set of those pairs $(\alpha, \beta)$ for which $\xi_{\alpha \beta}$ is defined.

We say that local action of tori is pure if $k_{\alpha}$ does not depend on $\alpha$.

Compare with definition 1.6 in [CG1].

Notations 0.2. Let us denote by $M^{(k)}$ the union of these $U_{\alpha}$ for which $k_{\alpha}$ is equal to an integer $k$. It is an open submanifold of $M$. If $m \in M^{(k)}$ then the orbit of $m$ (denoted $[m]_{k}$ ) of the local $T^{k}$ action is the orbit of $m$ of an action $\theta_{\alpha}$ such that $m \in U_{\alpha}$ and $k_{\alpha}=k$. According to definition $0.1[m]_{k}$ does not depend on $\alpha$. Let $X^{(k)}$ denote the orbit space of $M^{(k)}$ with the quotient topology, which is determined by transversal slices. (See the slice theorem [Br] II.4.4.)

Let us denote the quotient map $M^{(k)} \rightarrow X^{(k)}$ by $\varpi_{k}$. Let us denote a connected component of $M^{(k)}$ by $M_{i}^{(k)}$ where $i$ is a certain index. Set $M^{(k, l)}=M^{(k)} \cap M^{(l)}$ and $M^{(k, l, r)}=M^{(k)} \cap M^{(l)} \cap M^{(r)}$. If $m \in M^{(k, l)}, k \leq l$ then there is a map $\varpi_{l}^{k}$ sending $[m]_{k}$ to $[m]_{l}$ i.e. $\varpi_{l}=\varpi_{l}^{k} \varpi_{k}$.

Definition 0.3. The orbit space of a local action of tori with an atlas

$$
\left\langle\left\{U_{\alpha}\right\}_{\alpha \in \Lambda} ;\left\{\theta_{\alpha}\right\}_{\alpha \in \Lambda} ;\left\{\xi_{\alpha, \beta}\right\}_{(\alpha, \beta) \in \Lambda_{0}}\right\rangle
$$

is a collection of the orbit spaces $X^{(k)}$ together with projections $\varpi_{l}^{k}$ for $k \leq l$ such that $M^{(k, l)} \neq \emptyset$.

Let us denote by $\operatorname{Fix}_{i}^{(j)}$ the $i$ dimensional stratum of the fixed point set of $X^{(j)}$ and by $\operatorname{Fin}^{(j)}$ the subset of $X^{(j)}$ consisting of orbits with finite isotropy subgroups. Let $\operatorname{Pr}^{(j)}$ denote the set of principal orbits in $X^{(j)}$ and Cr the set of orbits in $X^{(1)}$ with isotropy subgroup isomorphic to $T^{1}$. 
A smooth structure on orbit spaces of compact group actions is discussed in [D]. The results can be adapted to local actions.

Assumption 0.4. We assume that the covering of $M$ by the open sets $U_{\alpha}$ is locally finite i.e. each compact set meets only a finite number of sets of the covering. Moreover we assume that the closures of the sets $U_{\alpha}, M^{(k)}, M^{(k, l)}$ and $M^{(k, l, r)}$ for all $\alpha, k, l$ and $r$ are manifolds with piece-wise smooth boundaries and that the corresponding pure tori actions can be extended to the closures. Let us denote the orbit spaces of the corresponding closures by $\operatorname{cl}\left(U_{\alpha} / T^{2}\right), \operatorname{cl}\left(X^{(k)}\right), \operatorname{cl}\left(X^{(k, l)}\right)$ and $\operatorname{cl}\left(X^{(k, l, r)}\right)$. Moreover we assume that the image of $M^{(k, l)}$ for any $k, l \operatorname{in} \operatorname{cl}\left(X^{(k)}\right)$ and in $\operatorname{cl}\left(X^{(l)}\right)$ is a collar neighbourhood of a part of the boundary.

We do not lose generality by starting from these assumptions since we can choose a subatlas of the maximal atlas of the local action of tori having the described properties.

Remark 0.5. The description of the strata given by isomorphism classes of isotropy subgroups in the 4-manifold with local action of tori is a consequence of the slice theorem for locally smooth actions of compact Lie groups on manifolds. The details can be found in $[\mathrm{Mi2}]$ or in $[\mathrm{R}]$.

Remark 0.6. According to the context $T^{2}$ will denote an acting torus (if we consider the local $T^{2}$ action on a connected component of $M^{(2)}$ ), a principal orbit (of the local action) or a singular chain (over $Z$ or $Z_{2}$ ) the orbit represents.

The inclusion of a principal orbit $T^{2}$ into $M$ induces a homomorphism:

$$
H_{*}\left(T^{2}, Z\right) \rightarrow H_{*}(M, Z) .
$$

Similarly for coefficients in $Z_{2}$.

One dimensional subgroups of the standard torus $T^{2}$ are described by primitive pairs of integers modulo multiplication by -1 . From now on according to the context we will use the symbol $(m, n)$, where $m, n$ are integers to denote

- a subgroup $T^{1}$ of $T^{2}$ or an affinely imbedded circle in $T^{2}$ with given orientation ( $m, n$ relatively prime).

- a singular chain or the homology class in the group $H_{1}\left(T^{2}, Z\right)$ the corresponding subgroup $T^{1}$ (as a submanifold of $T^{2}$ ) represents.

- an image in $C_{1}(M, Z)$ or in $H_{1}(M, Z)$ of the singular chain or the homology class of $H_{1}\left(T^{2}, Z\right)$ respectively (provided we have given the inclusion $T^{2} \hookrightarrow M$ ).

If $m, n$ are elements of $Z_{2},(m, n)$ will denote the singular cycle or the homology class in the group $H_{1}\left(T^{2}, Z_{2}\right)$ or its image in $H_{1}\left(M, Z_{2}\right)$. They come from integral singular cycles or integral homology classes.

Let $(m, n)$ and $\left(m^{\prime}, n^{\prime}\right)$ be singular cycles over $Z$ (in $T^{2}$ or in $\left.M\right)$. If $(m, n) \equiv$ $\left(m^{\prime}, n^{\prime}\right)(\bmod 2)$ then $(m, n)$ and $\left(m^{\prime}, n^{\prime}\right)$ are homologous as cycles over $Z_{2}$.

Remark 0.7. Let $x \in \operatorname{Pr}^{(2)}$. The group $\pi_{1}\left(X^{(2)}, x\right)$ acts on $H_{1}\left(T^{2}\right)\left(T^{2}\right.$ a principal fibre represented by $x$ ) and preserves the skew symmetric intersection form up to orientation. Thus we have a monodromy representation

$$
\pi_{1}\left(X^{(2)}, x\right) \rightarrow G L_{2}(Z) .
$$

Nonorientable monodromy corresponds to nonorientable loops. If we choose simple curves on the component of $X^{(2)}$ such that their complement is a disk then we can identify the fibre corresponding to any principal orbit over a point in the disk with the fibre over the base point, which can be identified with the acting torus $T^{2}$ 
provided we set a zero point in it. We will call such an identification a trivialization. Given a trivialization of the local action over some open simply connected set $U \subset \operatorname{Pr}^{(2)}$, some trivialization of the tangent space of $U$ (with smooth structure inherited from $M$ ) and canonical parallelization of $T^{2}$, we have a trivialization of the tangent space of $\varpi_{2}^{-1}(U) \cong U \times T^{2}$.

Definition 0.8. Let $M_{j}^{(i)}$ be a connected component of the set $M^{(i)}$. Let us choose a principal orbit $T^{i}$. A small equivariant tubular neighbourhood of the orbit admits the $T^{i}$ invariant parallelization given by the Lie algebra of $T^{i}$ and a base of the space tangent to a normal slice. If the section of the tangent $S O(4)$ bundle lifts to the section of the $\operatorname{Spin}(4)$ bundle we say that the local action is even. Otherwise we say that the local action is odd.

The spin structure on the orbit inherited from the spin structure on $M$ corresponds to the zero element of $H^{1}\left(T^{i}, Z_{2}\right)$ in the first case and to a non-zero element of $H^{1}\left(T^{i}, Z_{2}\right)$ in the second case.

Let us denote by $M_{\text {even }}^{(i)}$ (respectively $M_{\text {odd }}^{(i)}$ ) the sum of even (respectively odd) components of $M^{(i)}$.

Compare with the notions of even and odd circle action. (See for example [B].)

\section{THE MAIN THEOREM}

Theorem 1.1. Bordism group of spin 4-manifolds admitting local action of tori is isomorphic to $Z$. There is a generator with signature 16 .

We prove the theorem by reducing step by step the complexity of the local action on the manifold or topological complexity of the manifold.

First we show that $M^{(3)} \cup M^{(4)}$ and that $M_{\text {odd }}^{(1)}$ and $M_{\text {odd }}^{(2)}$ can be made empty without changing the spin bordism class of the local action of $M$. Then, without changing the manifold $M_{\text {even }}^{(1)}$, we change the local action so that the nonprincipal part of $M_{\text {even }}^{(1)}$ is included into $M_{\text {odd }}^{(2)}$.

Then we construct an example of a manifold with signature 16 with the property that $M^{(1)}=M_{\text {even }}^{(1)}$ and $X^{(1)}=\operatorname{Pr}^{(1)}$.

Assuming that the signature of the manifold is zero we show that by means of a spin bordism $M^{(1)}$ and $M^{(2)}$ can be made disjoined and both parts will admit even free local action.

Then using the fact that $\Omega_{3}^{\text {spin }}=0$ we show that $M^{(1)}$ is null spin cobordant.

Finally we reduce $M^{(2)}$ by means of a classifying map of the $T^{2}$ bundle.

$M^{(3)}$ and $M^{(4)}$ can be made empty.

\section{Lemma 1.2.}

1. Let $U \subset M^{(k)}$ be an open set. Let $V \subset \operatorname{cl}(V) \subset U \cap M^{(l)}$. There is a bordism $W$ such that $W=M \times[0,1]$ as a manifold, with product structure of local action in $(M-\operatorname{cl}(V)) \times[0,1]$ such that $V \times\{1\} \subset(M \times\{1\})^{(k)}-(M \times\{1\})^{(l)}$.

2. Let $U \subset M^{(k)}$ be an open set. Let $V \subset U$ be an open subset such that $U-V \subset$ $M^{(k, l)}$. There is a bordism $W, W=M \times[0,1]$ as a manifold, with product local action in $V \times[0,1]$, such that $(U-V) \times\{1\} \subset(M \times\{1\})^{(k)}-(M \times\{1\})^{(l)}$.

3. Let $N$ be a connected component of $M^{(k)}$ and $U \subset N$ an open $T^{k}$ invariant subset such that the local $T^{k}$ action is global on $U$. Let $V \subset U$ be a closed $T^{k}$ invariant subset. Let us assume that $N \cap M^{(i)}=\emptyset$ for $i \neq k$. Let $T^{l}<T^{k}$. 
Then there is a bordism $W, W=M \times[0,1]$ as a manifold, such that $U \times\{1\} \subset$ $(M \times\{1\})^{(l)},(N-V) \times\{1\} \subset(M \times\{1\})^{(k)}$ and $N \times\{1\} \cap(M \times\{1\})^{(i)}=\emptyset$ for $i \neq k, l$.

Proof. It suffices to define the sets $(M \times[0,1])^{(i)}, i=1,2,3,4$.

In (1) let us put

$$
\begin{aligned}
(M \times[0,1])^{(i)} & =M^{(i)} \times[0,1] \text { for } i \neq k, l, \\
((M-U) \times[0,1])^{(i)} & =(M-U)^{(i)} \times[0,1] \text { for } i=1,2,3,4, \\
U \times[0,1] \cap(M \times[0,1])^{(k)} & =U \times[0,1], \\
U \times[0,1] \cap(M \times[0,1])^{(l)} & =\left(U \cap M^{(l)}\right) \times[0,1 / 2) \cup(U-\operatorname{cl}(V)) \times[1 / 2,1] .
\end{aligned}
$$

In (2) let us put

$$
\begin{aligned}
(M \times[0,1])^{(i)} & =M^{(i)} \times[0,1] \text { for } i \neq l, \\
(U \times[0,1])^{(l)} & =U \times[0,1 / 2) \cup V \times[1 / 2,1] .
\end{aligned}
$$

In (3) let us put

$$
\begin{aligned}
(M \times[0,1])^{(i)} & =M^{(i)} \times[0,1] \text { for } i \neq k, l, \\
((M-N) \times[0,1])^{(i)} & =\left(M^{(i)}-N\right) \times[0,1] \text { for all } i, \\
(N \times[0,1])^{(k)} & =N \times[0,2 / 3) \cup(N-V) \times(1 / 3,1], \\
(N \times[0,1])^{(l)} & =U \times(1 / 3,1] .
\end{aligned}
$$

Lemma 1.3. A 4-manifold admitting local action of tori admits a spin cobordant local action of tori with the condition: $M^{(3)} \cup M^{(4)}=\emptyset$.

Proof. $M^{(4)}$ is a disjoined sum of several copies of $T^{4}$. According to 1.2 (1) we can assume that $M^{(4)} \cap\left(M^{(1)} \cup M^{(2)} \cup M^{(3)}\right)=\emptyset$. Then by $1.2(3)$ the action of $T^{4}$ on a component of $M^{(4)}$ can be replaced by means of bordism by $T^{1}$ action for some $T^{1}<T^{4}$ (we put $U=V=T^{4}$ ). Thus we may assume that $M^{(4)}=\emptyset$.

We can change the local action of tori on $M$ by bordism so that components of $M^{(3)}$ will be replaced by parts of $M^{(2)} \cup M^{(1)}$. If we forget the local action the bordism is the product $M \times[0,1]$.

The orbit space corresponding to a connected component $M_{j}^{(3)}$ of $M^{(3)}$ is homeomorphic to $(-1,1),[0,1), S^{1}$ or to $[0,1]$. In the last case up to a bordism (see 1.2 (1)) $M_{j}^{(3)} \cap\left(M^{(1)} \cup M^{(2)}\right)=\emptyset$. Moreover by means of a bordism (see $1.2(3)$ ) we may change the local action of tori so that the components lying over $[0,1]$ will be included in $M^{(1)}-\left(M^{(2)} \cup M^{(3)}\right)$.

If the orbit space of $M_{j}^{(3)}$ is $S^{1}$ then $M_{j}^{(3)}$ is a $T^{3}$ bundle over $S^{1}$. We first apply $1.2(1)$ in order to make $M_{j}^{(3)} \cap\left(M^{(1)} \cup M^{(2)}\right)=\emptyset$. Next we use 1.2 (3) for $k=3, l=1$, choosing $V$ and $U$ to be subsets lying over a closed and open interval in $S^{1}$. We may thus assume that the orbit space of $M_{j}^{(3)}$ is a subinterval of the $S^{1}$ homeomorphic to $(-1,1)$.

In the remaining cases the orbit space of $M_{j}^{(3)}$ is homeomorphic to $(-1,1)$ or to $[0,1)$. There is an $\epsilon>0$ so that the part of $M_{j}^{(3)}$ lying above $(-1,-1+\epsilon)$ in the 
first case or above $(1-\epsilon, 1)$ in both cases is included in $M^{(2,3)} \cup M^{(1,3)}$. Let us choose an orbit $p$ of the $T^{3}$ action belonging to one of the above intervals. Here $p \subset M_{j}^{(3)}$ or $p \in X_{j}^{(3)}$. If $p \subset M^{(1)}-M^{(2)}$, then there is a small neighbourhood $V_{p}$ of $p \in X_{j}^{(3)}$ such that $V_{p} \times T^{3} \subset M^{(1,3)}$. If $M^{(2)} \cap p \neq \emptyset$, then all components of this set correspond to the same two dimensional acting torus $T^{2}<T^{3}$, where $T^{3}$ is the acting torus on the component of $M^{(3)}$.

In fact a connected component of $M^{(2)} \cap p$ is equal to

$$
\{p\} \times(a, b) \times T^{1} \times T^{1} \subset\{p\} \times T^{3}
$$

for some interval $(a, b) \subset T^{1}$ (in appropriate local coordinates of $T^{1}$ ) and a suitable splitting: $T^{3}=T^{1} \times T^{1} \times T^{1}$. Any affine embedding of $T^{2}$ (an orbit of the $T^{2}$ action) into $T^{3}$ corresponding to another component of $M^{(2)} \cap p$ is of the form

$$
\left(t_{1}, t_{2}\right) \longmapsto\left(s_{1} t_{1}^{a_{1}} t_{2}^{b_{1}}, s_{2} t_{1}^{a_{2}} t_{2}^{b_{2}}, s_{3} t_{1}^{a_{3}} t_{2}^{b_{3}}\right) .
$$

If the first coordinate of this map is a surjective map onto $T^{1}$, then the second component of $M^{(2)} \cap p$ must intersect the first one. This is a contradiction. Thus the first coordinate is a constant map on the point $s_{1} \in T^{1}$ and other two are surjective. This means that the acting torus is the same as in the first component (orbits differ by translation in the first coordinate).

The similar property holds for all points in a small open neighbourhood $V_{p}$ of the point $p \in X_{j}^{(3)}$ with the same acting $T^{2}$. We can thus extend $M^{(2)}$ by bordism to the whole set $V_{p} \times T^{3}$ not changing $M^{(1)}$ and $M^{(3)}$.

Let us assume that $X_{j}^{(3)}=[0,1)$ and $V_{p}=(c, d) \subset(1-\epsilon, 1)$, where $V_{p}$ (as defined above) has one of the properties: $V_{p} \times T^{3} \subset M^{(1)}$ or $V_{p} \times T^{3} \subset M^{(2)}$. Let us denote the part of $M_{j}^{(3)}$ lying above $[0, c] \subset X_{j}^{(3)}$ by $M_{j, c}^{(3)}$ and above $[d, 1) \subset X_{j}^{(3)}$ by $M_{j, d}^{(3)}$. There is a bordism $W \cong M \times[0,1]$ (see 1.2) such that

$$
\begin{aligned}
M_{j, d}^{(3)} \times\{1\} \cap(M \times\{1\})^{(3)} & =M_{j, c}^{(3)} \times\{1\} \cap(M \times\{1\})^{(2)} \\
& =M_{j, c}^{(3)} \times\{1\} \cap(M \times\{1\})^{(1)}=\emptyset
\end{aligned}
$$

After applying similar argument in the case $X_{j}^{(3)}=(-1,1)$ and lemma 1.2 we can assume that a connected component of $M^{(3)} \cap\left(M^{(1)} \cup M^{(2)}\right)$ is equivariantly diffeomorphic to $(e, f) \times T^{3}$ for some small interval $(e, f) \subset X_{j}^{(3)}$ and is included in $\left(M^{(1)}-M^{(2)}\right) \cup\left(M^{(2)}-M^{(1)}\right)$.

In the case $X_{j}^{(3)}=[0,1)$ the component $M^{(1)}$ (or $M^{(2)}$ ) can be extended from $M^{(1)} \cap M_{j}^{(3)}$ (or $M^{(2)} \cap M_{j}^{(3)}$ respectively) to the whole component of $M^{(3)}$ by including the corresponding acting torus into $T^{3}$.

In the case $X_{j}^{(3)}=(-1,1)$ we can extend the components of $M^{(1,3)}$ or $M^{(2,3)}$ to parts over $(-1,-1 / 2)$ and $(1 / 2,1)$ respectively. If the acting tori at both ends are, let's say, $T_{1}^{1}$ and $T_{2}^{1}<T^{3}$ then over $(-2 / 3,2 / 3)$ we put a subgroup generated by these subgroups. If we have $T_{1}^{2}$ and $T_{2}^{2}$ then over $(-2 / 3,2 / 3)$ we put the intersection. If we have tori of different dimensions then over $(-2 / 3,0)$ put a torus of different dimension then over $(-1,-1 / 2)$ incident to it and over $(-1 / 3,2 / 3)$ as above the intersection or the group generated by the groups over $(-2 / 3,0)$ and $(1 / 2,1)$. The above changes can be depicted on $M^{(3)} \times[0,1]$ defining a bordism between the original manifold and the changed one. 
The trace of the bordism in each case is the product $M \times[0,1]$ which is obviously a spin manifold.

From now on we can make the

1.4 Assumption. $M^{(3)} \cup M^{(4)}=\emptyset$.

\section{REDUCTION OF THE ODD PART OF $M^{(2)}$}

Theorem 1.5. Let $M$ be a spin 4-manifold with a local action of tori then there exist a spin cobordant 4-manifold with a local action of tori, such that $X^{(2)}=\operatorname{Pr}^{(2)}$ and $M^{(2)}=M_{\text {even }}^{(2)}$.

Proof of Theorem 1.5. The proof uses a simple surgery trick.

1.6 Step 1 of the proof of Theorem 1.5. We can redefine the local action of tori in a small tubular neighbourhood of orbits with finite isotropy subgroup in $M^{(2)}$ by introducing a $T^{1}$ action for some $T^{1}<T^{2}$, where $T^{2}$ is the acting torus. The change can obviously be realized by a spin bordism.

1.7 Types of connected components of $M^{(2)}$. Take a connected component $M_{j}^{(2)}(j=1, \ldots, k)$ of $M^{(2)}$. There are three possibilities:

(1) $X_{j}^{(2)}=\operatorname{Pr}_{j}^{(2)}$. Then the boundary components of $\operatorname{cl}\left(X_{j}^{(2)}\right)$ correspond to connected components of $M^{(1,2)}$ and are homeomorphic to circles. The monodromy matrices corresponding to these circles (see 0.7 ) are parabolic because transition functions $\left(\xi_{\alpha \beta}\right.$ for $U_{\alpha}$ and $U_{\beta}$ intersecting with the corresponding component of $\partial\left(\operatorname{cl}\left(M_{j}^{(2)}\right)\right)$; see definition 0.1) preserve the acting torus $T^{1}$.

(2) $\mathrm{Cr}_{j} \cap M_{j}^{(2)} \neq \emptyset$ and $(0,1),(1,0)$ are pairs of integers modulo 2 represented by isotropy subgroups (with respect to certain trivialization).

(3) $\mathrm{Cr}_{j} \cap M_{j}^{(2)} \neq \emptyset$ and only the pair $(1,0)$ (modulo 2) is represented by isotropy subgroups (with respect to certain trivialization).

\subsection{Step 2 of the proof of Theorem 1.5.}

Lemma. Let $M$ be a spin manifold. Assume that $(p, q) \in H_{1}\left(T^{2}, Z_{2}\right)$ is represented by a one dimensional isotropy subgroup in some $M_{j}^{(2)}$ (according to certain trivialization). Then the spin structure restricted to a principal orbit in $M_{j}^{(2)}$ (with canonical parallelization) is determined by an element $c$ of $H^{1}\left(T^{2}, Z_{2}\right)$ such that

$$
\langle c,(p, q)\rangle=1
$$

In particular the local action is odd. Here $\langle\rangle:, H^{1}\left(T^{2}, Z_{2}\right) \times H_{1}\left(T^{2}, Z_{2}\right) \rightarrow Z_{2}$ is the usual pairing.

Proof. The regular tubular neighbourhood of the corresponding orbit with isotropy subgroup isomorphic to $T^{1}$ is diffeomorphic to $D^{2} \times S^{1} \times(-1,1)$. The principal $\operatorname{Spin}(4)$ bundle restricted to a loop $\partial\left(D^{2}\right) \times\{s\} \times\{t\},(s, t$ are fixed) with parallelization inherited from canonical parallelization on $\partial\left(D^{2}\right) \times S^{1} \times\{t\}$, corresponds to the image of a generator of $\pi_{1}(S O(2))$ under the map

$$
\pi_{1}(S O(2)) \rightarrow \pi_{1}(S O(4))=Z_{2}
$$

induced by inclusion $S O(2) \cong S O(2) \times\{1\}<S O(2) \times S O(2)<S O(4)$. Here $S O(2) \times S O(2) \cong T^{2}$ is a maximal torus of $S O(4)$. 


\subsection{Step 3 of the proof of Theorem 1.5.}

Lemma. Assume that all of the three elements:

$$
(0,1),(1,0),(1,1) \in H_{1}\left(T^{2}, Z_{2}\right)
$$

are represented by one dimensional isotropy subgroups in some $M_{j}^{(2)}$ (according to certain trivialization). Then $M$ is not spin.

Proof. It is a direct consequence of lemma 1.8 since we can find an open simply connected set $U \subset \operatorname{Pr}^{(2)}$ such that the representation of three types of orbits are included in $\operatorname{cl}(U)-U \subset$ Cr. See remark 0.7.

Here we can use the fact that the second Stiefel-Whitney class $w_{2} \in H^{2}\left(M, Z_{2}\right)$ is characterized by the property:

$$
w_{2} \cup c=c \cup c \text { for all } c \in H^{2}\left(M, Z_{2}\right) .
$$

Thus by Poincaré duality $M$ is spin iff $M$ is orientable and the intersection matrix over $Z_{2}$ is zero on the diagonal i.e. each 2-cycle over $Z_{2}$ has zero self-intersection. The proof, based on explicit construction of a 2-cycle with odd self-intersection, can be found in [Mi1].

The lemma in case of global $T^{2}$ actions corresponds to Theorem 3 in [P2] and Theorems 6.3 and 6.4 in $[\mathrm{Me}]$.

1.10 Step 4 of the proof of Theorem 1.5. Let $M_{j}^{(2)}$ be a component of $M^{(2)}$ of type 1.7 (2). If we take pairs of integers modulo 2 representing isotropy subgroups with respect to any trivialization then by lemma 1.9 exactly two kinds of them occur. We may assume that they are equal to $(1,0),(0,1) \bmod 2$.

1.11 Surgery tricks. Take a curve $\gamma$ in $X_{j}^{(2)}$ joining two points from $\mathrm{Cr}_{j}$. Let us assume that they correspond to isotropy subgroups representing the same element of $H_{1}\left(T^{2}, Z_{2}\right)$, according to certain trivialization over a dense open subset $U$ of $X_{j}^{(2)}$ such that $\gamma \subset U$. See 0.7. If such a curve does not exist, then according to section 4 in [P2] we can perform a surgery on $M$ near an orbit with isotropy subgroup isomorphic to $T^{1}$ in $M_{j}^{(2)}$ and obtain a manifold $M^{\prime}$ satisfying the assumption. The isotropy subgroups read off from the ends of the curve are let's say $(p, q),(r, s)$.

Let $N$ be a 4-manifold admitting a global $T^{2}$ action, being of type $1.7(2)$, with orbit space $Y$ homeomorphic to a disk and such that there exist two points in $\partial Y$ corresponding to isotropy groups $(p, q),(r, s)$ respectively. Here we use the description in [OR1] of the orbit spaces of 4-manifolds with $T^{2}$ action. Such a manifold $N$ exists in both cases: if $((p, q),(r, s)) \equiv((1,0),(1,0)) \bmod 2$ or $((p, q),(r, s)) \equiv((0,1),(0,1)) \bmod 2$. The argument uses the fact that $p / q$ and $r / s$ (in case $p \equiv r \equiv 1, q \equiv s \equiv 0$ ) can be written as a continued fraction

$$
b_{0}-\frac{1}{b_{1}-\frac{1}{\ddots \cdot-\frac{1}{b_{s}}}}
$$


with all entries even. This allows us to construct a sequence of pairs of relatively prime integers $\left(p_{i}, q_{i}\right)$ so that $\left(p_{0}, q_{0}\right)=(p, q),\left(p_{n}, q_{n}\right)=(1,0)$ and

$$
\operatorname{det}\left(\begin{array}{cc}
p_{i} & p_{i-1} \\
q_{i} & q_{i-1}
\end{array}\right)= \pm 1
$$

Similarly for $(r, s)$ we obtain $\left(r_{j}, s_{j}\right) ; j=1, \ldots, n^{\prime}$. The sought orbit space $Y$ will have the sequence

$$
\begin{gathered}
\left(p_{0}, q_{0}\right), \ldots,\left(p_{n}, q_{n}\right)=\left(r_{n^{\prime}}, s_{n^{\prime}}\right), \ldots,\left(r_{0}, s_{0}\right), \\
\left(r_{1}, s_{1}\right), \ldots,\left(r_{n^{\prime}}, s_{n^{\prime}}\right)=\left(p_{n}, q_{n}\right), \ldots,\left(p_{1}, q_{1}\right)
\end{gathered}
$$

as 1-dimensional isotropy subgroups data on the boundary.

The case $p \equiv r \equiv 0, q \equiv s \equiv 1$ is similar. The fact that this manifold is homeomorphic to a connected sum of several copies of $S^{2} \times S^{2}$ is proved in [OR1].

The surgery is as follows. Let us join two points in $Y$, with $(p, q)$ and $(r, s)$ as 1-dimensional isotropy subgroups data respectively, by a curve $\gamma^{\prime}$. Let us cut $X_{j}^{(2)}$ and $Y$ along the chosen curves $\gamma, \gamma^{\prime}$ and sew the corresponding parts so that the isotropy subgroups data agree at the ends of curves (according to chosen trivializations). If the orientations on $X_{j}^{(2)}$ and $Y$ are given in some neighbourhoods of $\gamma, \gamma^{\prime}$ respectively, then we glue the left side of $\gamma$ to the right side of $\gamma^{\prime}$ and the right side of $\gamma$ to the left side of $\gamma^{\prime}$. Let us denote the obtained surface by ${X^{\prime}}_{j}^{(2)}$.

$\gamma$ and $\gamma^{\prime}$ are chosen in such a way that according to the smooth structure on $X_{j}^{(2)}$ and $Y$ (see [D]) some neighbourhoods of $\gamma, \gamma^{\prime}$ are diffeomorphic.

The described cut and paste technique on the orbit space correspond to $T^{2}$ equivariant surgery on $M_{j}^{(2)} \cup N$. After the surgery we obtain a manifold $M_{j}^{\prime(2)}$ with the orbit space ${X^{\prime}}_{j}^{(2)}$. All 1-dimensional isotropy subgroups data on $\partial{X^{\prime}}_{j}^{(2)}$ are congruent to $(1,0)$ or to $(0,1) \bmod 2$ with respect to the trivializations induced on components of $X_{j}^{\prime(2)}$ from the trivialization on $X_{j}^{(2)}$.

The resulting component $M_{j}^{\prime(2)}$ (or sum of two components) is of type 1.7 (2) again. We can repeat the above surgery. It can be done in such a way that after the second and following steps of the surgery, components of the orbit space $X^{\prime(2)}$ have lower genus or have less boundary components. Thus after finitely many steps the monodromy representation corresponding to the obtained components of $M^{(2)}$ is trivial or preserves the $T^{1}$ subgroup corresponding to the nearby components of $M^{(1)}$.

We can make additional surgeries of the same type to ensure that any resulting component of $M_{j}^{\prime(2)}$ intersects with only one component of $M^{\prime(1)}$ in a connected set.

We can perform the same surgeries on all components of $M^{(2)}$ of type $1.7(2)$ and finally obtain a manifold $M^{\prime}$ such that any connected component of $M^{\prime(2)}$ of type 1.7 (2) admits global action of $T^{2}$. Now it is possible to redefine the local action of tori so that $M^{\prime(1)}$ will be extended onto components of $M^{\prime(2)}$ of type 1.7 (2). We also assume that these parts of $M^{\prime}$ are no longer in $M^{\prime(2)}$.

To make sure that the obtained manifold is spin, one should notice that according to lemma 1.8 there is a unique (inherited) spin structure on the small neighborhood of the 3-manifolds along which we cut and paste the manifolds. Moreover each of the above surgeries corresponds to a spin bordism. 
1.12 Step 5 of the proof of Theorem 1.5. Let $M_{j}^{(2)}$ be as in 1.7 (3). According to section 4 in [P2] we can perform a surgery on $M$ near an orbit in $M_{j}^{(2)}$ with isotropy subgroup isomorphic to $T^{1}$ and obtain a manifold $M^{\prime}$ such that the corresponding component $M^{\prime(2)}$ is of type 1.7 (2). The surgery corresponds to a spin bordism. Then we apply 1.11 .

1.13 Step 6 of the proof of Theorem 1.5. Let $M_{j}^{(2)}$ be as in 1.7 (1). Let us choose a principal orbit $T^{2}$ in $M_{j}^{(2)}$. The regular invariant tubular neighbourhood $\left(D^{2} \times T^{2}\right)_{1}$ inherits the spin structure from $M$. We can use natural trivialization of the tangent bundle of $M$ restricted to $T^{3}=\partial\left(D^{2} \times T^{2}\right)_{1}$, coming from the Lie algebra of $T^{2}$, tangent bundle of $\partial\left(D^{2}\right)$ and normal bundle of $\partial\left(D^{2} \times T^{2}\right)_{1}$ in $M$. With this trivialization the generator $\delta \in \pi_{1}\left(T^{3}\right)$ of the kernel of the homomorphism $\pi_{1}\left(T^{3}\right) \rightarrow \pi_{1}\left(\left(D^{2} \times T^{2}\right)_{1}\right)$ has the property that the principal Spin(4) bundle restricted to $\delta$ non-trivially covers the principal $S O(4)$ bundle. (Compare with 1.8.)

Let us assume that the local action is odd i.e. that there is a generator $\gamma$ of $\pi_{1}\left(T^{2}\right)=\pi_{1}\left(\left(D^{2} \times T^{2}\right)_{1}\right)$ such that the principal Spin(4) bundle restricted to $\gamma$ also non-trivially covers the principal $S O(4)$ bundle. Let $(p, q)$ denotes the corresponding subgroup of $T^{2}$.

Let us perform a surgery:

$$
M^{\prime}=\left(M-\left(D^{2} \times T^{2}\right)_{1}\right) \cup_{\partial}\left(T^{2} \times D^{2}\right)_{2}
$$

where $\left(T^{2} \times D^{2}\right)_{2}$ is the standard filling of $\left.T^{3}=\partial\left(D^{2} \times T^{2}\right)_{1}\right)$ in which the element $\gamma$ is trivial. The surgery corresponds to a spin bordism.

The spin structure on $M-\left(D^{2} \times T^{2}\right)_{1}$ inherited from $M$ extends to a spin structure on $M^{\prime}$. Moreover the local $T^{2}$ action on $M-\left(D^{2} \times T^{2}\right)_{1}$ extends to a local $T^{2}$ action on $M^{\prime}$ such that the corresponding component of $M_{j}^{\prime(2)}$ is of type 1.7 (3). Then we use 1.11 .

This completes the proof of Theorem 1.5.

\section{REDUCTION OF THE ODD PART OF $M^{(1)}$}

According to theorem 1.5 we can assume that $M^{(2)}=M_{\text {even }}^{(2)}$.

Lemma 1.14. Let us assume that the local action on a component $M_{j}^{(1)}$ of $M^{(1)}$ is odd. Then by means of a spin bordism we can get rid of $M_{j}^{(1)}$.

Proof. Let us observe that $M_{j}^{(1)} \cap M^{(2)}=\emptyset$ since $M^{(2)}$ consists only of even parts.

By a method described in [Fin1], [Fin2] we can introduce a local $T^{2}$ action extending the $T^{1}$ action in a neighbourhood of the set lying above $\operatorname{Fix}_{0}^{(1)} \cup \operatorname{Fin}^{(1)}$.

The intervals in the orbit space $X^{(1)}$, corresponding to orbits with finite isotropy groups, have ends in fixed points. In each fixed point at most two intervals meet. In this way we obtain several circles, several intervals and several isolated points. Let us choose smooth intervals in the principal part of $X^{(1)}$ joining the isolated points and ends of intervals. We can assume that near fixed points they agree with images of sets $\left(z_{1}, 0\right)$ or $\left(0, z_{2}\right)$ in some local coordinates, in which the action of $T^{1}$ is given by the formula

$$
t\left(z_{1}, z_{2}\right)=\left(t^{p} z_{1}, t^{q} z_{2}\right)
$$

for $|p|=1$ or $|q|=1$ respectively. 
We obtain closed circles in $X^{(1)}$. Over additional intervals we set isotropy subgroups equal to $Z_{1}$.

Near fixed points with given coordinates we can extend the $T^{1}$ action to the $T^{2}$ action by the formula.

$$
\left(t_{1}, t_{2}\right)\left(z_{1}, z_{2}\right)=\left(t_{1} z_{1}, t_{2} z_{2}\right) .
$$

Using the argument from [Fin1] and [Fin2] or a description of normal bundle to singular strata in [D] we can further extend the $T^{2}$ action to a local $T^{2}$ action on small tubular neighbourhood of the closure of the set of points with finite isotropy subgroups (including the intervals with $Z_{1}$ isotropy subgroup).

The boundary of the tubular neighbourhood is a disjoined sum of $T^{2}$ bundles over circles, denoted $N_{i}$. The monodromies of the bundles preserve $T^{1}$ (acting on $M^{(1)}$ ), so they are parabolic. Each $N_{i}$ bounds a $T^{1} \times D^{2}$ bundle $K_{i}$ over the circle, where $D^{2}$ corresponds to the eigenvalue of the monodromy matrix of $N_{i}$.

We separate the neighbourhood of the set lying above $\operatorname{Fix}_{0}^{(1)} \cup \operatorname{Fin}^{(1)}$ from the rest of the manifold by means of surgery:

$$
\left(M-N_{i} \times D^{1}\right) \cup_{\partial}\left(K_{i} \times S^{0}\right) .
$$

Here $N_{i} \times D^{1}$ is a neighbourhood of $N_{i}$ in $M$.

After a suitable change of the atlas we have obtained a disjoined sum of a manifold $M^{\prime(2)}$ with an odd local $T^{2}$ action without orbits with finite isotropy subgroups and a manifold $M_{j}^{\prime(1)}$ with an odd local $T^{1}$ action such that $\operatorname{Fix}_{0}^{(1)} \cup \operatorname{Fin}^{(1)}=\emptyset$.

The $S^{1}$ local action on $M_{j}^{\prime(1)}$ is semi-free and odd thus a similar argument as in [B] shows that it bounds a spin manifold with $S^{1}$ local action.

Applying cutting operations of 1.11 we can assume that the orbit space of any connected component of the manifold $M^{\prime(2)}$ is a disk. Then by [OR1] we see that the manifold $M^{\prime(2)}$ is equivariantly spin cobordant with disjoined sum of $S^{4}$ and thus equivariantly null spin cobordant.

\section{EVEN LOCAL ACTION ON $M^{(1)}$}

According to $1.5,1.14$ we may assume that $M_{\text {odd }}^{(1)}=M_{\text {odd }}^{(2)}=\emptyset$.

Remark 1.15. A component of $M_{\text {even }}^{(1)}$ can intersect only with $M_{\text {even }}^{(2)}$.

Let us introduce local $T^{2}$ action over a neighbourhood of $\operatorname{Fix}_{0}^{(1)} \cup \mathrm{Fin}^{(1)}$ as in 1.14. By changing the atlas we show that manifold is spin cobordant with a manifold $M$ being a sum of $M_{\text {even }}^{(1)}$ with free $T^{1}$ local action and $M^{(2)}$ with orbit space $X^{(2)}$ being a sum of annuli. Each annulus has one boundary component corresponding to $M^{(1,2)}$. We can choose a trivialization on an annulus and coordinates of the acting torus so that $(1,1)$ denotes the subgroup coming from the acting torus on $M^{(1)}$. Since the local action of $(1,1)$ is even the other boundary component of the annulus is marked with some isotropies equal to $(1,0),(0,1)$ modulo 2 with respect to the trivialization.

From now on we can make

1.16 Assumption. - All components of $M^{(2)}$ are of type 1.7 (1) with an even local action or are of type 1.7 (2) with an odd local action. The latter have no orbits with finite isotropy subgroup and intersect with $M^{(1)}$.

- Any connected component of $M^{(1)}$ admits even free local action. 
1.17 Example of a manifold with signature equal to 16. (1) Here we show an example of a compact spin 4-manifold having signature 16. This will give a counterexample to the Atiyah-Hirzebruch theorem for mixed local actions of tori. The manifold in the example represents a non-trivial generator of the spin bordism group.

(2) Let $R P^{1}$ denote the projectivization of the tangent space to $T^{2}$ in $\{1\} . R P^{1}$ is thus the set of 1-parameter subgroups in $T^{2}$. Let $H^{2}$ denote the upper half plane. The $R P^{1}$ bundle associated to the fibration $T^{2} \times H^{2}$ is trivial. It can be identified with the unit tangent bundle to $H^{2}$. Let $\mu$ be a vector tangent to $H^{2}$ having length 1 . Let us take the endpoint of the geodesic determined by $\mu$. It lies in the ideal boundary of $H^{2}$. In the upper half space model the ideal boundary is equal to $R \cup\{\infty\}$. The number (i.e. the endpoint of the geodesic) corresponds to the tangent of the angle between the 1-parameter subgroup corresponding to $\mu$ and the 1-parameter subgroup $(0,1)$, where the measurements are performed in the torus with conformal structure corresponding to $i \in H^{2}$. See [MS].

(3) Let $\xi$ be a smooth vector field in the upper half-plane $H^{2}$, invariant under the action of $P S L_{2}(Z)$, having zero in $i$ of index -1 and zero in $\frac{1+i \sqrt{3}}{2}$ of index +1 and pointing towards $\infty$ in a point $z$ with $\operatorname{Im}(z)>3 / 2$. Moreover we assume that $\xi$ has no other zeroes.

The vector field $\frac{\xi}{|\xi|}$ gives a section (outside the zero points) of the unit tangent bundle of $H^{2}$ and thus a section of the $R P^{1}$ bundle described above.

The standard action of $S L_{2}(Z)$ on $T^{2}$ induces the action of $P S L_{2}(Z)$ on $R P^{1}$. Thus the section is $S L_{2}(Z)$ invariant.

(3) Let $M_{1}^{(2)}$ be the total space of a $T^{2}$ bundle over an orientable surface $X_{1}^{(2)}$ with structure group $S L_{2}(Z)$. For any $T^{2}$ invariant metric on $M_{1}^{(2)}$ its restriction to a fibre gives a flat metric on $T^{2}$. If we assign to each fibre the conformal structure of its metric we obtain a well defined map

$$
\psi: X_{1}^{(2)} \rightarrow H^{2} / P S L_{2}(Z) .
$$

Let $i$ and $\frac{1+i \sqrt{3}}{2}$ also denote the image of the corresponding points in $H^{2} / P S L_{2}(Z)$.

The metric on $M_{1}^{(2)}$ can be chosen in such a way that $\psi$ has the property:

- For each $x \epsilon \psi^{-1}(i)$, (respectively $\left.x \epsilon \psi^{-1}\left(\frac{1+i \sqrt{3}}{2}\right)\right)$ there is a local smooth complex coordinate $z$ near $x$ and in the neighbourhood of $J(\psi(x))$ such that $J(\psi(z))=$ $z^{2}$ or $J(\psi(z))=\bar{z}^{2}\left(J(\psi(z))=z^{3}+1\right.$ or $J(\psi(z))=\bar{z}^{3}+1$ respectively).

Here $J$ is a modular function for $P S L_{2}(Z)$ having value $\infty, 0,1$ at the cusp, $i$ and $\frac{1+i \sqrt{3}}{2}$ respectively.

(4) Outside the isolated points $\psi^{-1}(i), \psi^{-1}\left(\frac{1+i \sqrt{3}}{2}\right)$ the vector field $\frac{\xi}{|\xi|}$ induces the section $\rho$ of the bundle over $X_{1}^{(2)}$ with fibre $R P^{1}$ associated to the $T^{2}$ fibration.

(5) Let $Y$ be the surface $H^{2} / \Gamma$, where $\Gamma$ is a subgroup of $P S L_{2}(Z)$ acting freely on $H^{2} . Y$ inherits an orientation from $H^{2}$. There is a metric on $H^{2} \times_{\tilde{\Gamma}} T^{2}(\tilde{\Gamma}$ a lift of $\Gamma$ to $S L_{2}(Z)$ which will be chosen below in (10)) such that the corresponding map

$$
\psi: Y \rightarrow H^{2} / P S L_{2}(Z)
$$

is the canonical projection

$$
H^{2} / \Gamma \rightarrow H^{2} / P S L_{2}(Z) .
$$


(6) Let $\Gamma$ be a subgroup of $P S L_{2}(Z)$ acting freely on $H^{2}$ such that $H^{2} / \Gamma$ is a punctured torus (i.e. homeomorphic to $T^{2}-D^{2}$ ), where the cusp corresponds to the matrix $-a^{6}$ for

$$
a=\left(\begin{array}{ll}
1 & 1 \\
0 & 1
\end{array}\right)
$$

Let $X_{1}^{(2)}$ be a surface constructed from 4 copies, $Y_{1}, \ldots, Y_{4}$, of the surface $\bar{H}^{2} / \Gamma$. Here $\bar{H}^{2}$ denotes the compactified version of $H^{2}$ realized by cutting off open horoballs $P S L_{2}(Z)$ congruent to the horoball $\left\{z \in H^{2}: i m(z)>2\right\} . Y_{1}, \ldots, Y_{4}$ are joined together by performing connected sums of their boundaries so that the orientations on the copies are compatible and thus give an orientation on $X_{1}^{(2)}$. We may assume that the subset of $Y_{i}(i=1,2,3,4$ respectively) corresponding to the complement of horoballs $P S L_{2}(Z)$ congruent to the horoball $\left\{z \in H^{2}: i m(z)>3 / 2\right\}$ is canonicaly identified with a subset $X_{i}$ of $X_{1}^{(2)}$. In other words the connected sums are performed in the region corresponding to $\left\{z \in H^{2}: i m(z)>3 / 2\right\}$. We can assume further that the connected sums are performed near the lines of right-angled tori. More precisely the construction of the connected sum of $\partial\left(Y_{i}\right)$ and $\partial\left(Y_{j}\right)$ goes as follows.

Let us cut $Y_{i}$ and $Y_{j}$ along one of the intervals corresponding to $\left\{z \in H^{2}\right.$ : $r e(z)=0 ; 2 \geq i m(z) \geq 7 / 4\}$. Let us glue $Y_{i}$ and $Y_{j}$ along the boundary so that the corresponding coordinates agree and the resulting piecewise smooth surface is connected and has orientation compatible with the previous one. Near the new saddle point (a point corresponding to $7 i / 4$ ) we can define the smooth structure and the map into $H^{2}$ using local complex variable $w=z-7 i / 4$ :

$$
w \longmapsto w^{2} \longmapsto \psi\left(w^{2}\right)
$$

The smooth structure and the map can be extended to the whole surface so that it agrees with smooth structure and the map $\psi$ outside some neighbourhood of the saddle point.

In other words we replace the trivial covering $\psi$ over some neighbourhood of $7 i / 4$ by a branched covering.

(7) Let $M_{1}^{(2)}$ be the total space of the $T^{2}$ bundle constructed from 4 copies of $\bar{H}^{2} \times_{\tilde{\Gamma}} T^{2}$, joined correspondingly (see (6)) in such a way that the monodromy along $\partial\left(X_{1}^{(2)}\right),\left(X_{1}^{(2)}\right.$ is the base space), is equal to $a^{24}$.

By the above assumptions the map $\left.\psi\right|_{X_{i}}$ is the canonical projection of $X_{i}(i=$ $1,2,3,4)$ on $H^{2} / P S L_{2}(Z)$ and $\psi$ maps $X_{1}^{(2)}-\bigcup_{i} X_{i}$ into the subset of $H^{2} / P S L_{2}(Z)$ corresponding to $\operatorname{Im}(z)>3 / 2$. Moreover $\psi$ restricted to small neighbourhood of $\partial\left(X_{1}^{(2)}\right)$ is a covering preserving orientation and $\partial\left(X_{1}^{(2)}\right)$ maps onto the circle in $H^{2} / P S L_{2}(Z)$ represented by the horocycle $\{z: \operatorname{Im}(z)=2\}$.

(8) We can calculate that the number of points in $\psi^{-1}(i)$ is equal to $3 \times 4=12$ and in $\psi^{-1}\left(\frac{1+i \sqrt{3}}{2}\right)$ to $2 \times 4=8$. The resulting index coming from $\xi$ is -4 . There is a simple contractible curve $\gamma$ in $X_{1}^{(2)}$ bounding a disk $D^{2}$ such that all points of $\psi^{-1}(i) \cup \psi^{-1}\left(\frac{1+i \sqrt{3}}{2}\right)$ lie in $D^{2}$.

(9) Let $\eta$ be the gradient of a Morse function on $X_{1}^{(2)}$ having its maximum on $\partial\left(X_{1}^{(2)}\right)$ and critical points in $\operatorname{int}\left(X_{1}^{(2)}\right)$. Since $X_{1}^{(2)}$ has genus 4 we can assume that $\eta$ has 9 zero points. We can assume that all of them lie in $D^{2}$. 
(10) Let us choose standard free generators $\alpha_{i}, \beta_{i}(i=1,2,3,4)$ of $\pi_{1}\left(X_{1}^{(2)}\right)$ and corresponding simple closed curves $a_{i}, b_{i}$ in $X_{1}^{(2)}$ disjoined from $D^{2}$. We assume that $\alpha_{i}, \beta_{i}$ comes from $X_{i}$. The map $\psi$ gives a representation

$$
\pi_{1}\left(X_{1}^{(2)}\right) \rightarrow P S L_{2}(Z) .
$$

We can choose a lift to $S L_{2}(Z)$ of the representation (see (5)) in such a way that over $a_{i}, b_{i}$ the section $\rho$ of the $R P^{1}$ bundle over $X_{1}^{(2)}$ associated to the $T^{2}$ fibration has a lift to the section $\tilde{\rho}$ of the $S^{1}$ bundle tangent to fibers of the $T^{2}$ fibration.

Thus over a small neighbourhood of the sum of loops $a_{i}, b_{i}$ (in fact over the complement of $D^{2}$ ) the total space of the $T^{2}$ fibration has a $T^{2}$ invariant framing compatible with the splitting into vertical and horizontal subbundles. It is determined by $\rho$ and $\eta$. Let us choose the spin structure on this part of the manifold so that the section of the $S O(4)$ bundle lifts to the section of the Spin(4) bundle.

(11) Let us split the disk $D^{2}$ into two smaller ones $D^{2}=D_{1}^{2} \cup D_{2}^{2}$ such that $D_{1}^{2}$ consists of all points coming from the singularities of $\xi$ (contributing total index -4 in case of the $R P^{1}$ bundle or total index -2 in case of the $S^{1}$ bundle) and 8 critical points of $\eta . D_{2}^{2}$ consists of the remaining single critical point of $\eta$.

The framing determined by $\rho$ and $\eta$ can be extended over $D_{1}^{2} \times T^{2}, T^{2}$ invariantly, since the section of the $S O(4)$ bundle over $\partial\left(D_{1}^{2}\right) \times T^{2}$ with respect to the trivialization $D_{1}^{2} \times T^{2}$ represents trivial element of $\pi_{1}(S O(4))$.

There is a trivial $\operatorname{Spin}(4)$ bundle covering the trivial $S O(4)$ bundle over $D_{1}^{2} \times T^{2}$. Here we take canonical $T^{2}$ invariant trivialization of the tangent bundle of $D_{1}^{2} \times T^{2}$. The section of the $S \operatorname{pin}(4)$ bundle over $\partial\left(D_{1}^{2}\right) \times T^{2}$, covering the section of the $S O(4)$ bundle, determined above, can be extended over $D_{1}^{2} \times T^{2}$.

Let us extend the spin structure accordingly.

(12) We can assume that $D_{2}^{2}$ has a common interval with $\partial\left(X_{1}^{(2)}\right)$ and that $X_{1}^{(2)}-D_{2}^{2}$ is a smooth surface diffeomorphic with $X_{1}^{(2)}$. Thus the part of the manifold lying over $X_{1}^{(2)}-D_{2}^{2}$ (denoted $N_{1}$ ) is diffeomorphic with $M_{1}^{(2)}$.

The spin structure constructed above on $N_{1}$ induces the spin structure on the boundary $\partial\left(N_{1}\right)$ i.e. on the $T^{2}$ bundle over $S^{1}$ with monodromy $a^{24}$.

The section $\eta$ over $\partial\left(X_{1}^{(2)}-D_{2}^{2}\right)$ of the tangent $S O(2)$ bundle induces via the diffeomorphism a section over $\partial\left(X_{1}^{(2)}\right)$ differing from the trivial section $\eta$ on $\partial\left(X_{1}^{(2)}\right)$ by a single twist.

Thus the spin structure on $\partial\left(N_{1}\right)$ according to the framing inherited (via the diffeomorphism) from that on $\partial\left(M_{1}^{(2)}\right)$ (i.e. given by the sections $\rho$ and $\eta$ ) corresponds to the element of $H^{1}\left(\partial\left(N_{1}\right)\right)$ which is nontrivial on the horizontal loop, covering once the base $S^{1}$ of the $T^{2}$ fibration, and is trivial on vertical loops.

(13) If $T^{1}$ is a subgroup of $T^{2}$ preserved by the automorphism $a$ then $T^{1}$ acts on $\partial\left(N_{1}\right)$ and this action is free and even. $\partial\left(N_{1}\right)$ is the total space of the $T^{1}$ bundle over $T_{1}^{2}$ with Euler number 24. According to the argument given in [B] $T_{1}^{2}:=\partial\left(N_{1}\right) / T^{1}$ inherits a spin structure. It corresponds to the non-zero element of $H^{1}\left(T_{1} ; Z_{2}\right)$ according to canonical parallelization of $T_{1}^{2}$.

(14) Let us take 12 copies of the 4-manifold constructed in [CG1] 1.9. Monodromies along three loops in the boundary are conjugate to $a^{2}, a^{2},-a^{2}$. We may assume that the corresponding 1-dimensional isotropy subgroups are equal to $(1,0) ;(0,1) ;(1,1)$. Let us remove a tubular neighbourhood of the stratum with isotropy subgroup $(1,1)$ in each copy. The resulting boundary in each copy is a 
$T^{2}$ bundle over $S^{1}$ with monodromy conjugate to $-a^{2}$ and preserving $(1,1)$. The obtained manifold is spin.

Let us join the copies by performing a connected sum on the orbit spaces of their boundaries, similarly as in (6). The obtained manifold (denoted $N_{2}$ ) is also spin, with the boundary diffeomorphic to $\partial\left(N_{1}\right)$. (Here the monodromy of the $T^{2}$ bundle preserves $(1,1)$.) The action of $(1,1)$ near the boundary is even while the local action of $(1,0)$ and $(0,1)$ (with respect to some trivialization) is odd in any spin structure inherited from $\mathrm{N}_{2}$.

We may choose a spin structure on $N_{2}$ so that with respect to the canonical trivialization on $\partial\left(N_{2}\right)$, given by the splitting into vertical and horizontal parts and by the eigenvector of the monodromy matrix, it is given by an element of $H^{1}\left(\partial\left(N_{2}\right)\right)$ acting nontrivially on the horizontal loop covering once the base of the bundle.

$\partial\left(N_{2}\right)$ is a $T^{1}$ bundle over $T_{2}^{2}$ with the Euler number 24. Similarly as in (13) we see that $T_{2}^{2}:=\partial\left(N_{2}\right) / T^{1}$ inherits a spin structure. It corresponds to the non-zero element of $H^{1}\left(T_{2}^{2} ; Z_{2}\right)$ according to canonical parallelization of $T_{2}^{2}$.

(15) The $T_{1}^{2}, T_{2}^{2}$ are spin cobordant where the trace of the bordism is a product. We just identify $T_{1}^{2}$ with suitable orientation with $T_{2}^{2} \times\{0\}$ while $T_{2}^{2}$ is identified with $T_{2}^{2} \times\{1\}$. The identifications are chosen in such a way that the spin structures on the boundary of the product are inherited from a spin structure on the product. Using again the argument given in [B] we obtain a $T^{1}$ equivariant spin bordism between $\partial\left(N_{1}\right)$ and $\partial\left(N_{2}\right)$ covering the bordism of the orbit spaces.

Let us denote the trace of the bordism by $N_{3}$. There is a $T^{1}$ action given by fibers of the $T^{1}$ fibration.

(16) Let us choose a suitable orientation on $N_{1}$ and glue the manifold with $N_{3}$. Then let us glue the resulting manifold with $N_{2}$. The gluing maps correspond to identifications given in (15).

The obtained manifold $N$ admits local action of tori given by that on $N_{i}$. It is closed and admits a spin structure.

Applying residue formulas (see e.g. in $[R],[Y]$ ) or related result in [Mi1] we easily find that each component corresponding to isotropy subgroup isomorphic to $T^{1}$ contributes $2 / 3$ to the signature. The rest of the manifold $N$ admits a polarization. We thus obtain

$$
\operatorname{sign}(N)=2 \times 12 \times \frac{2}{3}=16 .
$$

Remark 1.18. Given a compact spin 4-manifold $a$ with local action of tori we can add some multiplicity of the manifold constructed in the example in order to make the signature of the resulting manifold equal to zero.

Let us observe that since each component of $M^{(1)}$ admits an even local action we can perform a sequence of adjacent connected sums along orbits of $M^{(1)}$ (connected sums on the level of orbit spaces) in order to make $M^{(1)}$ connected. The same procedure can be applied to even and odd parts of $M^{(2)}$ and the corresponding parts of $M^{(1,2)}$ respectively. The operations correspond to spin bordism.

If the orbit space of a component of $M_{\text {odd }}^{(2)}$ has genus $g>0$, then by the procedure described in the proof of 1.5 we can lessen the genus. If the corresponding part of $M^{(1,2)}$ is connected we can assume that the orbit space of the component of $M_{\text {odd }}^{(2)}$ is an annulus.

From now on we make the: 
1.19 Assumption. $-\operatorname{sign}(M)=0$.

- $M^{(1)}, M_{\text {even }}^{(2)}, M_{\text {odd }}^{(2)}$ and the corresponding parts of $M^{(1,2)}$ are connected.

- If $M_{\text {odd }}^{(2)} \neq \emptyset$, then its orbit space is homeomorphic to an annulus.

\section{SEPARATION $M^{(1)}$ FROM $M^{(2)}$}

Lemma 1.20. Let $\gamma$ be a simple loop in the orbit space of $M_{\text {even }}^{(2)}$ with monodromy I. If $\gamma$ bounds a compact submanifold of $X^{(2)}$ then the equivariant surgery on $M$ corresponding to killing the homotopy class of $\gamma$ in the orbit space is a spin surgery.

Proof. The part of $M$ lying over the loop $\gamma$ is diffeomorphic to $T^{3}$. If $\gamma$ bounds a compact submanifold of $X^{(2)}$, then the $T^{3}$ bounds a spin manifold with signature zero since $M_{\text {even }}^{(2)}$ admits a polarization and $\eta$ invariant of $T^{3}$ is zero. Thus the Rohlin invariant of the spin structure on $T^{3}$ is equal to zero. The spin structure on $T^{3}$ thus corresponds to an non-zero element $c$ of $H^{1}\left(T^{3} ; Z_{2}\right)$. Since the local action is even $c$ acts non-trivially on the generator of $H_{1}\left(T^{3} ; Z_{2}\right)$ corresponding to $\gamma$. Thus the surgery

$$
\left(M-T^{2} \times \gamma \times D^{1}\right) \cup_{\partial}\left(T^{2} \times D^{2} \times S^{0}\right)
$$

is a spin surgery.

Proposition 1.21. A spin 4-manifold with local actions of tori and signature zero is cobordant with a manifold such that $M^{(3)} \cup M^{(4)}=\emptyset$. Moreover $M^{(1)} \cap M^{(2)}=\emptyset$ and $M^{(1)}$ and $M^{(2)}$ admit free even local actions.

Proof. (1) $M^{(3)} \cup M^{(4)}=\emptyset$ was proved in 1.3 .

(2) According to 1.16 and 1.19 we can assume that $M_{\text {even }}^{(2)}$ is connected and that the corresponding part of $M^{(1,2)}$ is also connected. We can further assume that $M_{\text {odd }}^{(2)}$ is connected and has no orbits with finite isotropy subgroups. Moreover the corresponding part of $M^{(1,2)}$ is diffeomorphic to a tubular neighbourhood of a $T^{2}$ bundle over a circle. The monodromy of the bundle preserves $T^{1}$ (acting on $M^{(1)}$ ), so it is parabolic. We take a local trivialization so that the torus $T^{1}$ represents the 1-cycle $(1,1)$ over $Z_{2}$.

$X_{o d d}^{(2)}$ is homeomorphic to an annulus with one boundary component corresponding to boundary and the other is (according to the trivialization) marked with some isotropies equal to $(1,0),(0,1)$ modulo 2 . The monodromy modulo 2 preserves the pair $\{(1,0),(0,1)\}$ since it preserves $(1,1)$.

The number of fixed points is even since $\chi(M)=\operatorname{sign}(M)=0 \bmod 2$.

Let us denote the fixed points by $x_{1}, \ldots, x_{2 k}$ according to the cyclic order on the component of $\partial\left(X_{o d d}^{(2)}\right)$. Let us perform a $T^{2}$ equivariant connected sum for each pair $x_{2 i-1}, x_{2 i}(i=1, \ldots, k)$. We obtain the manifold $M^{\prime}$ such that $M_{\text {odd }}^{\prime(2)}$ has monodromy in $\Gamma_{2}$, the group consisting of matrices from $S L_{2}(Z)$ equal to the identity modulo $2 . M_{\text {odd }}^{\prime(2)}$ has no fixed points and its orbit space is a sphere with holes.

Let us observe that there are three conjugacy classes of parabolic subgroups in $\Gamma_{2}$, namely the groups generated by $a^{2}, b^{2}$ and $\left(a^{2} b^{2}\right)^{-1}$ where

$$
a=\left(\begin{array}{ll}
1 & 1 \\
0 & 1
\end{array}\right), \quad b=\left(\begin{array}{cc}
1 & 0 \\
-1 & 1
\end{array}\right) .
$$


The monodromy matrices along components of $\mathrm{Cr}$ are up to multiplication by $\pm I$ conjugated in $\Gamma_{2}$ to some powers of $a^{2}$ or $b^{2}$ since they preserve $(1,0)$ or $(0,1)$ modulo 2. The monodromy matrix corresponding to $M^{(1,2)}$ is up to multiplication by $\pm I$ conjugated to some power of $\left(a^{2} b^{2}\right)^{-1}$ since it preserves $(1,1)$. Since $\operatorname{sign}(M)=0$, according to residue formulas, the total contribution to the signature coming from $\mathrm{Cr}$ has to be zero. It implies that the local degree of a regular classifying map:

$$
X_{\text {odd }}^{(2)} \rightarrow H^{2} / \Gamma
$$

(compare $1.17(3)$ ) is zero. See [Mi1] for details. Thus the monodromy matrix corresponding to $M^{(1,2)}$ is $\pm I$. This makes it possible to separate (as in 1.14) the corresponding part of $M^{\prime(2)}$ from $M^{\prime(1)}$ by introducing isotropy subgroup $T^{\prime 1} \cong$ $(1,0) \bmod 2$ such that $T^{1} \cap T^{1}=\{1\}$ along a loop in $\varpi_{2}\left(M^{(1,2)}\right)$ and changing the atlas. Here $T^{1}$ denotes the torus acting on $M^{\prime(1)}$. The resulting part with $T^{1}$ local action will admit free $T^{1}$ local action. The odd part $M^{\prime \prime}{ }_{\text {odd }}^{(2)}$ is null spin cobordant. See 1.11, [OR1] and 1.14.

(3) If $M^{(1)}$ and $M^{(2)}$ admit free even local actions and $M^{(1)}, M^{(2)}$ and $M^{(1,2)}$ are connected, then the monodromy along the loop $\gamma$ in $X^{(2)}$ corresponding to $M^{(1,2)}$ is equal to the identity. It means that $\partial M^{(1)}$ is a trivial $T^{1}$ bundle over $T^{2}$. The proof goes as follows.

The double cover $\tilde{M}^{(1)}$ of $M^{(1)}$ corresponding to the orientation double cover $\tilde{X}^{(1)}$ of $X^{(1)}$ admits a circle action. In case the covering of $\partial M^{(1)}$ is not connected the orientations on both sheets coincide. In particular the Euler numbers of $T^{1}$ bundles are equal. There is a classifying map

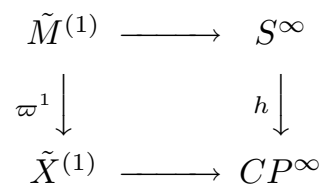

which maps orbits to fibers of the Hopf fibration. $\tilde{M}^{(1)}$ is 4 - and $\tilde{X}^{(1)}$ is 3dimensional so the map can be changed by a fiber preserving homotopy to a map having its image in the total space of the Hopf fibration $S^{3} \rightarrow C P^{1}$. Boundary of $\tilde{X}^{(1)}$ consists of tori which map to $C P^{1}$ with total degree 0 . It follows from the functoriality of the exact sequence of a pair for $\left(C P^{1}, D^{3}\right)$ and $\left(\tilde{X}^{(1)}, \partial \tilde{X}^{(1)}\right)$.

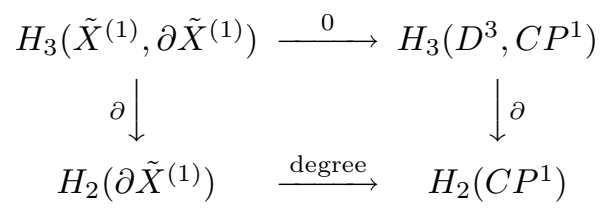

Thus the monodromy along the loop $\gamma$ in $X^{(2)}$ corresponding to $M^{(1,2)}$ is equal to $\pm I$. $-I$ can not occur since it can not be written as $\Pi_{i}\left[A_{i}, B_{i}\right]$ for $A_{i}, B_{i} \in S L_{2}(Z)$ or as $\Pi_{i} C_{i}^{2}$ for $C_{i} \in G L_{2}(Z)$.

Applying lemma 1.20 we can kill the homotopy class of $\gamma$ in $X^{(2)}$ by equivariant spin surgery on $M$. Then by a change of the atlas we can make $M^{(1,2)}=\emptyset$.

FREe, EVEN LOCAL ACTION ON $M^{(1)}$

Assuming that $M^{(1)}$ is connected we have two possibilities. Either $M^{(1)}$ is a principal $T^{1}$ bundle over a spin 3-manifold or $M^{(1)}$ is a non-orientable $T^{1}$ bundle 
over a non-orientable 3-manifold with pin $^{-}$structure. This follows from an argument similar to that in [B] Proposition 2.2. If we are given an $S^{1}$ invariant metric, then the bundle tangent to $M^{(1)}$, denoted $\tau\left(M^{(1)}\right)$, splits as $\tau \oplus \operatorname{det} \tau$, where $\tau$ is the $R^{3}$ subbundle orthogonal to fibers and $\operatorname{det} \tau$, the determinant line bundle of $\tau$, is isomorphic to the $R^{1}$ bundle tangent to fibers. The spin structure on $\tau\left(M^{(1)}\right)$ induces an $S^{1}$ invariant pin ${ }^{-}$structure on $\tau$. See $[\mathrm{KT}]$. Let $\tau\left(X^{(1)}\right)$ be the tangent bundle to $X^{(1)}$. It is isomorphic to $\tau / S^{1}$. It inherits the pin ${ }^{-}$structure.

Lemma 1.22. $M^{(1)}$ admitting free even local action being an orientable $T^{1}$ bundle over a spin 3-manifold is null spin cobordant

Proof. By means of a surgery we change the bundle into a trivial one.

Let us choose a triangulation of $X^{(1)}$. In each 3-simplex $\triangle^{3}$ of the triangulation of $X^{(1)}$ take the 1 -skeleton $\triangle_{1}^{3}$ of the dual cell complex. It consists of four intervals joining the middle point of the simplex with the middle points of its 2-dimensional faces. The $S^{1}$ bundle over $X$ is isomorphic to the $S^{1}$ bundle, which when restricted to any 3-simplex $\triangle^{3}$ is trivial in $\triangle^{3}-\triangle_{1}^{3}$. To each interval in $\triangle_{1}^{3}$ we may associate an integer describing "transversal local Euler number" (it tells how many times local section of the $S^{1}$ bundle over the link of the interval wraps along the fibre when the link approaches a point on the interval). The sum of these integers over the four intervals is zero (the $S^{1}$ bundle over the boundary of the 3 -simplex is trivial). Let $D$ be the subcomplex of the 1-skeleton of the dual complex consisting of those intervals to which a non-zero integer is assigned. If we take the assigned numbers into account then $D$ gives a 1-cycle $d$ representing an element of $H_{1}(X, Z)$. Let $d$ be the obstruction class to existence of a global section of the $T^{1}$ bundle (i.e. the first Chern class). $d$ is homologous to a 1-cycle supported in a disjoined sum of smoothly embedded circles $c$ in $X^{(1)}$.

Choosing a suitable framing along $c$ we can perform a spin surgery on $X^{(1)}$ killing the homotopy class of each component of $c$. The surgery corresponds to equivariant surgery on $M^{(1)}$. After the surgery the obstruction class is zero.

Then since $\Omega_{3}^{\text {spin }}=0$ we see that the total space of the trivial $S^{1}$ bundle is null spin cobordant.

Lemma 1.23. $M^{(1)}$ admitting free even local action being a non-orientable $T^{1}$ bundle over a pin ${ }^{-}$3-manifold is null spin cobordant

Proof. Let $V$ be an orientable surface in $X^{(1)}$ representing homology class dual to the first Stiefel-Whitney class of $X^{(1)}$. First by a method similar to that in the orientable case we show that the obstruction cycle is supported in disjoined sum of smoothly embedded circles transversal to $V$. The 1-cycle $d$ represents an element of $H_{1}(X, \tilde{Z})$, where $\tilde{Z}$ is a locally constant coefficient system with stalk $Z$ and holonomy in $O(1)$ given by orientation sheaf on $X^{(1)}$. Then we can kill components of the obstruction cycle to existence of a global section which lie in $X^{(1)}-V$ in the same way as in orientable case.

A component of the obstruction cycle intersecting $V$ with "transversal Euler number" $k$ has a tubular neighbourhood in $X^{(1)}$ diffeomorphic to $D^{2} \times S^{1} / Z_{2}$. The $T^{1}$ bundle over the neighbourhood is diffeomorphic to $D^{2} \times S^{1} \times T^{1} / Z_{2}$, where $Z_{2}$ acts by $\epsilon(d, s, t)=\left(\bar{d},-s, t^{-1}\right)$. The trivialization of the $T^{1}$ bundle outside the neighbourhood determines a section on the boundary. There is a $T^{2}$ local action on $D^{2} \times S^{1} \times T^{1} / Z_{2}$ coming from the standard $T^{2}$ action on $D^{2} \times T^{1}$. The base space is an annulus with one boundary component marked with isotropy subgroup $(-k, 1)$. 
The section of the $T^{1} \cong(1,0)$ subbundle over the second boundary component is given by orbits of the subgroup $(0,1)$ intersecting the section of the $T^{2}$ bundle. The local action of $(-k, 1)$ is odd and the local action of $(1,0)$ is even. Thus the local action of $(0,1)$ is odd and we can cut off the neighbourhood of the stratum corresponding to the isotropy subgroup $(-k, 1)$ by introducing over the second boundary component the isotropy subgroup $(0,1)$. It is an analogous surgical operation to that in 1.14 .

The resulting component of $M_{\text {odd }}^{(2)}$ is equivariantly null spin cobordant and the resulting $T^{1}$ bundle has global section.

Since $\Omega_{3}^{\text {pin- }}=0$ by $[\mathrm{KT}]$ we see as in orientable case that the total space of the $S^{1}$ bundle is null spin cobordant.

\section{Free, EVEN LOCAL ACTION ON $M^{(2)}$}

$M^{(2)}$ is the total space of a $T^{2}$ bundle over $X^{(2)}$ with structure group $\operatorname{Aff}\left(T^{2}\right)$. If we choose a $T^{2}$ invariant metric on $M^{(2)}$ and assign to each fibre the conformal structure of its metric, we then obtain a well defined map

$$
\psi: X^{(2)} \rightarrow H^{2} / P S L_{2}(Z)
$$

in case $X^{(2)}$ is orientable and

$$
\psi: X^{(2)} \rightarrow H^{2} / P G L_{2}(Z)
$$

in case $X^{(2)}$ is non-orientable. In this case the map locally lifts to $H^{2} / P S L_{2}(Z)$ provided we have chosen an orientation on $X^{(2)}$ and on a fibre $T^{2}$. In both cases $\psi$ locally lifts to $H^{2}$. This map determines $M^{(2)}$ up to a choice of the class being the obstruction to a global section.

Definition 1.24. We will call a map between surfaces stable if it has the property: when the derivative does not have the maximal rank the only singularities are cusps and folds.

In local coordinates the map near singularity is of one of the forms:

$$
\left(x_{1}, x_{2}\right) \longmapsto\left(x_{1}, x_{2}^{2}\right) ;\left(x_{1}, x_{2}\right) \longmapsto\left(x_{1}, x_{1} x_{2}+x_{2}^{3}\right)
$$

for fold and cusp respectively.

Applying the transversality theorem of $\mathrm{H}$. Whitney we obtain

Lemma 1.25 (Regularity of a classifying map). Let us assume that $X^{(2)}$ is orientable. In case $X^{(2)}$ is non-orientable the statement is true locally.

There is a map $\psi^{\prime}$ in a small neighbourhood of $\psi$ with the following properties:

(1) For each $x \epsilon \psi^{-1}(i)$, respectively $x \epsilon \psi^{-1}(e(1 / 6))$, there is a local smooth complex coordinate $z$ near $x$ and in the neighbourhood of $J(\psi(x))$ such that

$$
\begin{gathered}
J(\psi(z))=z^{2} \text { or } J(\psi(z))=\bar{z}^{2}, \\
J(\psi(z))=z^{3}+1 \text { or } J(\psi(z))=\bar{z}^{3}+1,
\end{gathered}
$$

respectively.

(2) $J(\psi)$ is stable in $X$ outside a small neighbourhood of $\psi^{-1}(i)$ and $\psi^{-1}(e(1 / 6))$. Moreover the curve $\psi^{-1}\{i y: y \geq 1\}$ is disjoined from singularities of cusp type and is transversal to the curve given by singularity of fold type. 
Here $J$ (as in 1.17) is a modular function for $P S L_{2}(Z)$ having value $\infty, 0,1$ at the cusp, $i$ and $\frac{1+i \sqrt{3}}{2}$ respectively.

Reduction of $X^{(2)}$. The curve $\psi^{-1}\{i y: y \geq 1\}$ consists of simple piecewise smooth loops. We can omit loops, which are contractible. The curve divides $X^{(2)}$ into connected submanifolds such that over each the $T^{2}$ bundle has monodromy in the group conjugated to the stabilizer $D_{6}$ of $e(1 / 6)$ in $G L_{2}(Z)$.

We can picture $X^{(2)}$ as

$$
\bigcup X_{i} / \sim
$$

where $X_{i}$ are surfaces which are the result of cutting the surface $X^{(2)}$ along a smooth simple curves homotopic with loops in $\psi^{-1}\{i y: y \geq 1\}$. $\sim$ denotes the corresponding gluing mappings.

Let $\delta_{i}$ be a simple closed curve in $X_{i}$ such that $\delta_{i}$ and $\partial\left(X_{i}\right)$ bound an orientable surface with genus zero. The monodromy along $\delta_{i}$ is equal to $\Pi_{i}\left[A_{i}, B_{i}\right]$ for certain orientable elements $A_{i}, B_{i}$ of $D_{6}$ if $X_{i}$ is orientable, and to $\Pi_{i} A_{i}^{2}$ for certain nonorientable elements of $D_{6}$ if $X_{i}$ is nonorientable. Thus in both cases it is equal to $I$.

Applying lemma 1.20 we can perform a surgery killing the homotopy class of $\delta_{i}$.

If a neighbourhood of a loop $\gamma$ in $X_{i}$ is non-orientable then the square of the monodromy matrix along $\gamma$ is $I$. Thus by lemma 1.20 we can kill the homotopy class of $\gamma^{2}$.

If a neighbourhood of a loop $\gamma^{\prime}$ in $\psi^{-1}\{i y: y \geq 1\}$ is non-orientable then the square of the monodromy matrix along $\gamma$ is $I$. Similarly using lemma 1.20 we can kill the homotopy class of $\gamma^{\prime 2}$.

After the surgeries we obtain:

(1) some $T^{2}$ bundles over $R P^{2}$,

(2) some $T^{2}$ bundles over orientable surfaces $X_{i}^{\prime}$ with monodromy in $D_{6}$ and

(3) some $T^{2}$ bundles over orientable surfaces $Y_{j}$, which consist of the curves corresponding to orientable part of $\psi^{-1}\{i y: y \geq 1\}$.

(1) The monodromy along the nontrivial loop in $R P^{2}$ has an integer eigenvector thus we can change the atlas so that the $T^{2}$ bundle will be included in $M_{\text {even }}^{(1)}$.

(2) $T^{2}$ bundle with monodromy in $D_{6}$ over an orientable surface $X_{i}^{\prime}$ of genus $g$ can be then surgered (using lemma 1.20) into $g$ disjoined $T^{2}$ bundles with monodromy in $D_{6}$ over $T^{2}$. The surgery corresponds to killing $g-1$ loops in the surface $X_{i}^{\prime}$ homologous to zero. Since $D_{6} \cap S L_{2}(Z)$ is isomorphic to $Z_{6}$ we can find a basis of $H_{1}\left(T^{2}\right)$ in which the monodromy matrices of a $T^{2}$ bundle are equal to $(A, I)$. If we can kill the loop with trivial monodromy we include the resulting manifold into $M_{\text {even }}^{(1)}$ by choosing a $T^{1}<T^{2}$. If we can not kill the loop with trivial monodromy then we can assume that the obstruction class to existence of a global section is zero. If it is not we separate a suitable $T^{2}$ bundle over $S^{2}$ by means of a surgery reversed to connected sum of the base spaces. If the Euler class is zero we can introduce a new direction of an action obtaining local $T^{3}$ action. The manifold has a compatible $T^{1}$ even action coming from the new direction. Thus by means of a change of the atlas we include the manifold into $M_{e v e n}^{(1)}$.

(3) The $T^{2}$ bundles over orientable surfaces $Y_{j}$, which consist of the curves corresponding to the orientable part of $\psi^{-1}\{i y: y \geq 1\}$, (in case $Y_{j}$ have genus $g>1$ ) are built from $T^{2}$ bundles over $S_{k} \cong S^{2}-\left(D_{1}^{2} \cup D_{2}^{2} \cup D_{3}^{2}\right)\left(D_{1}^{2}, D_{2}^{2}, D_{3}^{2}\right.$ are disjoined 
smoothly imbedded disks in $S^{2}$ ) with boundary monodromies $\pm I$ by means of a suitable gluing mappings. By means of lemma 1.20 we can kill all loops (among the boundary loops of $S_{k}$ ) which are homologous to zero in $Y_{j}$ and have monodromy I. If three boundary loops in $S^{2}-\left(D_{1}^{2} \cup D_{2}^{2} \cup D_{3}^{2}\right)$ have monodromy $I$, then at least one loop can be killed. We can thus assume that at least one boundary loop in $S^{2}-\left(D_{1}^{2} \cup D_{2}^{2} \cup D_{3}^{2}\right)$ has monodromy $-I$.

Let us assume that two different surfaces $S_{k_{1}}, S_{k_{2}}$ are glued along the common loop $\delta$ with monodromy $-I$. Then in the resulting sphere with four boundary components two have monodromy $I$. We can find a third loop with monodromy $I$. Then we apply the above remark.

Let us assume that two different surfaces $S_{k_{1}}, S_{k_{2}}$ are glued along the common loop $\delta_{1}$ with monodromy $I$. Let us assume that the third surface $S_{k_{3}}$ is glued to $S_{k_{2}}$ along the common loop $\delta_{2}$ with monodromy $-I$. Then as above in the resulting sphere with five boundary components we can find a loop along which the cutting is possible.

Thus we can assume that at most two different surfaces $S_{k_{1}}, S_{k_{2}}$ are glued together. In this case we can find a loop homologous to zero with monodromy $I$ and then apply lemma 1.20.

After the sequence of surgeries and changes of atlases the manifold $M^{(2)}$ consists of a disjoined sum of

- $T^{2}$ bundles over $T^{2}$,

$-T^{2}$ bundles over $S^{2}$.

We can change the atlas on $T^{2}$ bundles over $S^{2}$ and include them in $M_{\text {even }}^{(1)}$. In case of a $T^{2}$ bundle over $T^{2}$ we can find a basis of $H_{1}\left(T^{2}\right)$ such that the corresponding monodromy matrices are equal to $(A, \pm I)$. Let us express $A=A_{1} \ldots A_{n}$ as a product of parabolic matrices. Then by a sequence of local connected sums on the base we change the base torus into a surface of genus $n$ such that the monodromy matrices along the base loops are equal to $\left(A_{1}, \pm I, \ldots, A_{n}, \pm I\right)$. Then using lemma 1.20 we change the surface into $n$ tori, having monodromy matrices equal to $\left(A_{i}, \pm I\right)$. Since the monodromy groups have integer eigenvalues we can include the manifolds into $M_{\text {even }}^{(1)}$.

\section{REFERENCES}

[AH] M.F.Atiyah and F.Hirzebruch, "Spin-manifolds and group actions in Essays on Topology and Related Topics" Springer-Verlag, Berlin (1970), 18-28. MR 43:4064

[B] L.D.Borsari, "Bordism group of semi-free circle actions on Spin manifolds", Trans.AMS 301 (1987), 479-487. MR 88g:57037

[Br] Glen E. Bredon, "Compact transformation groups," Academic Press New York, (1972). MR 54:1265

[CFG] J.Cheeger, K.Fukaya, M.Gromov, "Nilpotent structures and invariant metrics on collapsed manifolds" J.AMS 5 (1992), 327-372. MR 93a:53036

[CG1] J.Cheeger and M.Gromov, "Collapsing Riemannian Manifolds While Keeping Their Curvature Bounded I," J.Diff. Geom. 23 (1986), 309-346. MR 87k:53087

[CG2] J.Cheeger and M.Gromov, "Collapsing Riemannian Manifolds While Keeping Their Curvature Bounded II," J.Diff. Geom. 32 (1990), 269-298. MR 92a:53066

[D] M.W.Davis, "Smooth G-manifolds as collection of fibre bundles" Pacific J. Math. 77/2 (1978). MR 80b:57034

[G] M.Gromov, "Volume and bounded cohomology," Publ. IHES, 56 (1983), 213-307. MR 84h:53053

[KT] R.C.Kirby, L.R.Taylor, "Pin structures on low-dimensional manifolds." London Math. Soc. Lecture Note Ser., 151 177-242, Cambridge Univ. Press, Cambridge, 1990. MR 94b:57031 
[Me] P.Melvin, "On 4-manifolds with Singular Torus Actions," Math. Ann. 256 (1981), 255276. MR 82m:57027

[Mi1] Piotr Mikrut, "Local $T^{2}$ actions on compact 4-manifolds" Thesis, Polish Academy of Sciences, Warsaw.

[Mi2] Piotr Mikrut, "Bordism of 4-manifolds with T-structure" preprint.

[MS] John W. Milnor, James D. Stasheff, "Characteristic classes" Princeton, New Jersey (1974). MR 55:13428

[OR1] Peter Orlik, Frank Raymond, "Actions of the torus on 4-manifolds I," Trans. AMS 152 (1970), 531-559. MR 42:3808

[OR2] Peter Orlik, Frank Raymond, "Actions of the torus on 4-manifolds II," Topology 13 (1974), 89-112. MR 50:1274

[P1] Peter Sie Pao, "The topological structure of 4-manifolds with effective torus actions .I" Trans. AMS 227 (1977), 279-317. MR 55:4232

[P2] Peter Sie Pao, "The topological structure of 4-manifolds with effective torus actions .II" Ill. J.Math. 21 (1977), 883-894. MR 56:13241

[R] X. Rong, "The existence of polarized F-structures on volume collapsed 4-manifolds" Geometric and Functional Analysis 3/5 (1993). MR 95a:53063

$\mathrm{Y}] \quad$ D. G. Yang, "A residue theorem for secondary invariants of collapsing Riemannian manifolds",Ph.D Thesis, State University of Stony Brook (1986).

[Fin1] Ronald Fintushel, "Circle actions on simply connected 4-manifolds," Trans.AMS 230 (1977). MR 56:16639

[Fin2] Ronald Fintushel, "Classification of circle actions on 4-manifolds," Trans.AMS 242 (1978). MR 81e:57036

Mathematical Institute, University of Wroceaw, Pl.Grunwaldzki $2 / 4, \quad 50-384$ Wroclaw, Poland

E-mail address: mikrut@math.uni.wroc.pl 